\title{
Abnormal apical-to-basal transport of dietary ovalbumin by secretory IgA stimulates a mucosal Th1 response
}

\author{
J Abed $^{1,2}$, C Lebreton ${ }^{1,2}$, G Champier ${ }^{3}$, A Cuvillier ${ }^{3}$, M Cogné $^{4}$, B Meresse $^{1,2}$, C Dugave $^{5}$,

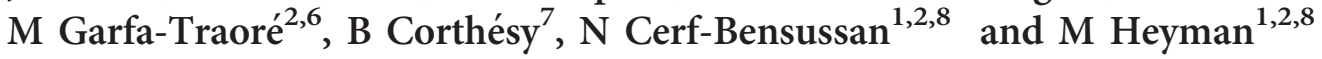

In celiac disease, enhanced permeability to gliadin peptides can result from their apico-basal transport by secretory immunoglobulin A1 (SlgA1) binding to the CD71 receptor ectopically expressed at the gut epithelial surface. Herein, we have established a mouse model in which there is apico-basal transport of the model antigen ovalbumin (OVA) by specific SIgA1 and have analyzed local T-cell activation. Transgenic DO11.10 mice were grafted with a hybridomasecreting OVA-specific humanized IgA1, which could bind mouse CD71 and which were released in the intestinal lumen as SIgA. CD71 expression was induced at the gut apical surface by treating the mice with tyrphostin A8. Following gavage of the mice with OVA, OVA-specific CD4 ${ }^{+} \mathrm{T}$ cells isolated from the mesenteric lymph nodes displayed higher expression of the activation marker CD69 and produced more interferon gamma in mice bearing the hybridomasecreting OVA-specific IgA1, than in ungrafted mice or in mice grafted with an irrelevant hybridoma. These results indicate that the protective role of SIgA1 might be jeopardized in human pathological conditions associated with ectopic expression of CD71 at the gut surface.

\section{INTRODUCTION}

In the normal intestine, a small fraction of undigested dietary peptides can be taken up by the epithelium, but they are largely degraded within the lysosomal compartment of enterocytes and may thereby loose their antigenicity. ${ }^{1}$ An increased translocation of intact gliadin peptides is, however, observed across the duodenal mucosa in active celiac disease (ACD). ${ }^{2}$ Paracellular leakage has been suggested as a possible explanation ${ }^{3}$ as paracellular permeability is increased in ACD patients, as evidenced by lactulose/mannitol permeability tests, ${ }^{4,5}$ by ultrastructural modifications of tight junctions, ${ }^{6}$ and by lower electrical resistance and increased mannitol fluxes in duodenojejunal biopsies. ${ }^{78}$ However, tight junctional complexes show size selectivity and the leak of small inert markers (lactulose, $342 \mathrm{Da}$; mannitol, $182 \mathrm{Da}$ ) does not accurately reflect epithelial permeability to gliadin peptides that are much larger in size $(2000-4000 \mathrm{Da}){ }^{8}$ Conversely, several studies point to an increased transcellular transport of gliadin peptides in ACD. ${ }^{2,8-11}$ We have demonstrated in ACD that gliadin peptides complexed to specific secretory immunoglobulin A (SIgA) can undergo mucosal-to-serosal transcellular transport via the CD71 receptor. CD71 is best known as a high-avidity receptor for transferrin but has more recently been shown to be a low-avidity receptor for human polymeric IgA1 (pIgA1). ${ }^{12,13}$ Using polarized Caco-2 cell monolayers, we have observed that SIgA1 binds to apically expressed CD71 and enters the recycling pathway, thus avoiding degradation in lysosomes and instead undergoing rapid translocation to the basolateral membrane. ${ }^{14}$ Accordingly, in the presence of specific SIgA, gliadin peptide p31-43 is rapidly transported across the epithelial monolayer and delivered partly intact into the subepithelial compartment. Similarly, Rauhavirta et al. ${ }^{15}$ have observed that celiac IgA can promote the apical-to-basal transcellular transport of intact gliadin peptides through

\footnotetext{
${ }^{1}$ Institut National de la Santé et de la Recherche Médicale (INSERM), UMR989, Paris, France. ${ }^{2}$ Université Paris Descartes-Sorbonne Paris Cité, Institut IMAGINE, Paris, France. ${ }^{3} \mathrm{~B}$ Cell Design, rue C. Legendre, Limoges, France. ${ }^{4} \mathrm{CNRS}$, UMR6101 Université de Limoges, Limoges, France. ${ }^{5}$ Commissariat à l'Énergie Atomique, iBiTecS, Service d'Ingénierie Moléculaire des Protéines, Gif-sur-Yvette, France. ${ }^{6}$ IFR 94, Imagery platform, Paris, France and ${ }^{7}$ R\&D Laboratory, Division of Immunology and Allergy, University State Hospital (CHUV), Lausanne, Switzerland. Correspondence: N Cerf-Bensussan (nadine.cerf-bensussan@inserm.fr)

${ }^{8} \mathrm{NC}-\mathrm{B}$ and $\mathrm{MH}$ are co-last authors.
} 
cultured polarized epithelial Caco-2 cells. This mechanism cannot operate in the normal intestine as CD71 is mainly confined to the basolateral membrane of crypt enterocytes. Therefore, IgA are transported by the receptor for polymeric immunoglobulins (pIgR) from the lamina propria into the lumen, where they form complexes with luminal antigens and oppose their absorption. In ACD, however, due to overexpression and redistribution of CD71 from the basolateral to the apical surface of enterocytes, the protective role of SIgA is distorted and SIgA-gliadin complexes can be retro-transported toward the lamina propria. ${ }^{12}$ It is suspected that this enhanced absorption of IgA/gliadin complexes promotes intestinal T-cell activation, but this has not yet been demonstrated. This possibility deserves consideration as recent work has shown that, in vivo, retrieval of luminal antigens complexed to IgG via the FcRn receptor could stimulate mucosal $\mathrm{CD} 44^{+}$T-cell responses. ${ }^{16,17}$ Herein, we describe the establishment of a mouse model to study SIgA-dependent protected transport of the dietary protein ovalbumin (OVA) and show that it can enhance the activation of cognate mucosal $\mathrm{CD} 4{ }^{+} \mathrm{T}$ helper type 1-biased cells.

\section{RESULTS}

\section{Human IgA, but not murine IgA, bind murine CD71}

Binding of IgA to the murine transferrin receptor CD71 was first assessed by flow cytometry in the intestinal epithelial MODE K and ICcl2 cell lines, which both strongly expressed this receptor (Figure 1a, middle panel and not shown, respectively). No binding of murine $\mathrm{pIgA}$ to either MODE K (Figure 1a, left panel) or ICcl2 cells (not shown) was observed. In contrast, strong binding of human SIgA to both cell lines was detectable (Figure 1a, right panel and not shown, respectively), suggesting that human SIgA, which is known to bind human CD71, might also bind murine CD71. Transfection of MODE $\mathrm{K}$ cells by CD71-specific small interfering RNA (siRNA) simultaneously reduced murine CD71 expression and membrane binding of human SIgA compared with MODE K cells transfected with a control siRNA (Figure 1a, right panel). Furthermore, interactions between murine CD71 and human SIgA could be demonstrated using the probe ligation assay Duolink. Thus, labeling of CD71 and pre-bound human SIgA gave rise to red dots on the surface of untransfected or control siRNA-transfected MODE K cells (not shown and Figure $\mathbf{1 b}$ left panel, respectively), indicating that they were interacting within a $40-\mathrm{nm}$ distance. Moreover, this staining was markedly lower in MODE K cells transfected with CD71-specific siRNA (Figure 1b, right panel). Taken together, these results indicate that human SIgA but not mouse pIgA binds to mouse CD71, an observation that can be explained, as discussed below, by the strong homology between mouse and human CD71 and the distinct structures of mouse IgA and human IgA1.

\section{CD71 expression is upregulated by tyrphostin A8 (TyrA8) in murine enterocytes}

In humans, mucosal-to-serosal transepithelial transport of SIgA requires the expression of CD71 on the apical side of enterocytes. ${ }^{12,14}$ As TyrA8 is known to enhance CD71mediated apical-to-basal transcytosis of insulin-transferrin complexes in Caco-2 cells and in diabetic rats, ${ }^{18,19}$ expression of CD71 in the intestine of mice treated with TyrA8 for $48 \mathrm{~h}$ was examined by confocal microscopy (Figure 2). In contrast to
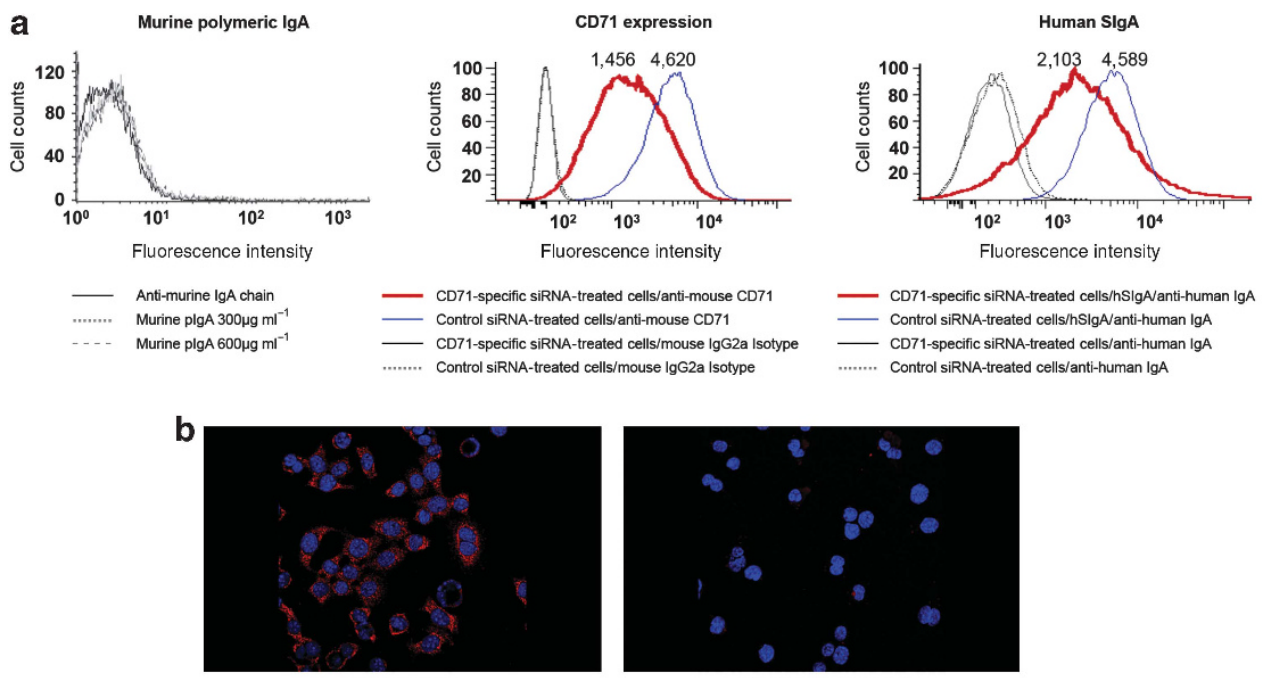

Control siRNA

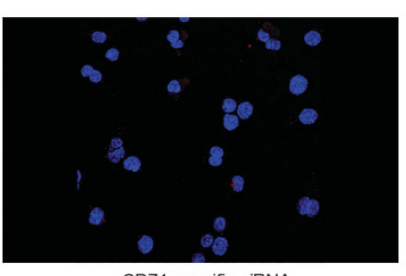

CD71-specific siRNA

Duolink-PLA staining on MODE $\mathrm{K}$ cells

Figure 1 Human secretory immunoglobulin $\mathrm{A}(\mathrm{hSIgA})$ but not murine polymeric $\lg \mathrm{A}(\mathrm{plg} \mathrm{A})$ bind murine CD71. (a) Flow cytometry analysis of binding of murine plgA (left panel) or of $\mathrm{hSIgA}$; $\left(300 \mathrm{~g} \mathrm{ml}^{-1}\right.$; right panel) and CD71 expression (middle panel) in the murine MODE K intestinal epithelial cell line, untransfected or transfected with CD71-specific small interfering RNA (siRNA; red bold line) or control siRNA (blue line). Results for one representative experiment out of 3-4 independent experiments are shown. Median fluorescence intensities are indicated. (b) Analysis by probe ligation assay Duolink and confocal microscopy of interactions between hSIgA and CD71 in MODE K cells transfected with control siRNA (left panel) or CD71-specific siRNA (right panel). Red dots indicate where CD71 and SIgA molecules interact within a 40-nm distance. Results for one representative experiment out of two independent experiments, each performed in triplicate, are shown. 
a

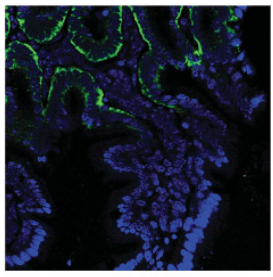

Untreated Balb/c

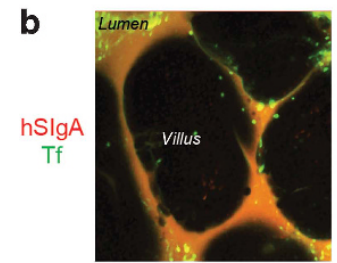

Untreated Balb/c

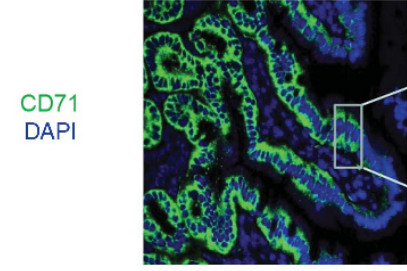

TyrA8-treated Balb/c
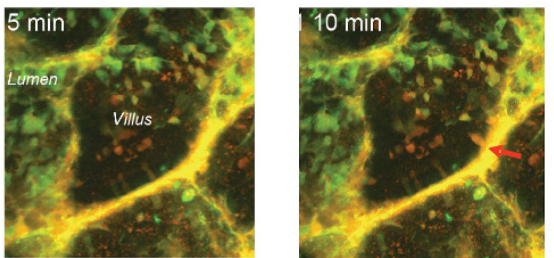

TyrA8-treated Balb/c
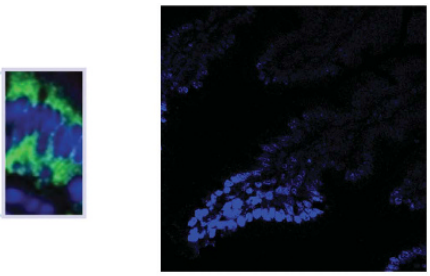

TyrA8-treated Balb/c Isotype control

Figure 2 Mucosal-to-serosal epithelial transport of transferrin (Tf) and human secretory immunoglobulin A (hSIgA) in tyrphostin A8-treated mice. (a) Comparison by confocal microscopy of CD71 expression (green staining) in duodenal sections from Balb/c mice either untreated (left panel) or $48 \mathrm{~h}$ after intraperitoneal treatment with tyrphostin A8 (TyrA8). Cell nuclei are stained in blue with DAPI (4,6-diamidino-2-phenylindole). One representative set of images out of five comparable observations is shown. (b) Analysis by multiphotonic video microscopy of apical binding and transcellular transport of Tf (green) and human SIgA (red) in duodenal segments from Balb/c mice either untreated (left panel) or 48 h after TyrA8 treatment (right panels). In TyrA8treated mice, images are shown at 5-, 10-, and 20-min time points after the intraluminal injection of Tf and SIgA. Image of a duodenal segment from a control mouse at the 20-min time point is shown. Red arrows highlight co-localizing hSIgA and Tf entering enterocytes. One representative set of images out of four comparable experiments is shown (see also Supplementary movies online).

control mice in which CD71 was confined to crypt enterocytes, TyrA8-treated mice also expressed CD71 in the villous epithelium both at the apical and basal poles of enterocytes (Figure 2a). To confirm that CD71 was accessible from the luminal side after TyrA8 treatment, FITC (fluorescein isothiocyanate)-labeled transferrin was injected into the lumen of ligatured duodenal segments and followed up by multiphotonic video microscopy (Figure 2b). Binding of transferrin (green) to the apically expressed CD71 receptor and its rapid mucosal-to-serosal transepithelial transport was observed in the intestine from TyrA8-treated mice but not in control mouse intestine. Moreover, we observed that human SIgA labeled with a red fluorescence probe could be transported simultaneously with transferrin from the apical to the basal side of enterocytes in TyrA8-treated but not in control mice (see the extensive co-localization of transferrin and SIgA during transfer observed as the yellow/orange staining in Figure $\mathbf{2 b}$ and Supplementary movies online). This simultaneous transfer, compatible with previous evidence that human IgA1 and transferrin bind to distinct sites on CD71, ${ }^{20}$ supports the notion that apically expressed murine CD71 enables the retro-transport of human SIgA across the mouse duodenum.

\section{Intact OVA is transported in intestinal biopsies of mice carrying a backpack (BP) tumor secreting OVA-specific humanized IgA1 (hzlgA1)}

The inability of mouse IgA to bind to murine CD71 precluded the setting up of an in vivo model of CD71-IgA-mediated transport in wild-type mice. We therefore turned to $\alpha 1 \mathrm{KI}$ mice, which produce chimeric hzIgA. Flow cytometry analysis of MODE K cells transfected with CD71-specific or control siRNA and multiphotonic video microscopy of duodenal segments showed that epithelial binding and transport of phzIgA were comparable to that of human SIgA (Figure 3a,b; see Supplementary movies online), suggesting that a pertinent model might be set up using $\alpha 1 \mathrm{KI}$ mice. In a first set of experiments, $\alpha 1 \mathrm{KI}$ mice were immunized by systemic and oral administration of OVA. They were then treated with TyrA8 for $48 \mathrm{~h}$ and the intestinal transport of ${ }^{3} \mathrm{H}$-OVA was assessed in Ussing chambers. As shown in Figure 4a (left panel), a small increase in the percentage of OVA transported in intact form was observed in TyrA8-treated OVA-immunized mice $(18.5 \% \pm 18.4)$ compared with TyrA8-treated naive mice (Control) $(8.0 \% \pm 9.6, P=0.066)$, but this increase did not reach statistical significance. Accordingly, fluxes of intact OVA were not significantly different in TyrA8-treated OVA-immunized $\alpha 1 \mathrm{KI}$ mice $\left(0.66 \pm 0.77 \mathrm{pmol} 90 \mathrm{~min}^{-1} \mathrm{~cm}^{-2}\right)$ and control mice $\left(0.26 \pm 0.40 \mathrm{pmol} 90 \mathrm{~min}^{-1} \mathrm{~cm}^{-2}, P=0.132\right.$; Figure 4a, right panel). Notably however, if significant titers of OVA-specific hzIgA were detected in the serum of immunized $\alpha 1 \mathrm{KI}$ mice (Figure 3c), these antibodies remained below the threshold of detection in the intestinal fluid (not shown). Therefore, it is likely that their concentration was insufficient to allow IgA-dependent transport.

In order to increase the amount of OVA-specific IgA in the intestinal lumen, we used an alternative model in which mice were grafted with hybridoma cells secreting hzIgA specific for OVA or for the control antigen $\beta$-lactoglobulin. Two weeks after injection, mice exhibited visible tumor on their back (BP mice). Although serum concentrations of total hzIgA were significantly lower in BP mice (median: $610 \mu \mathrm{g} \mathrm{ml}^{-1}$ ) than in OVA-immunized $\alpha 1 \mathrm{KI}$ mice (median: $1,603 \mu \mathrm{g} \mathrm{m}^{-1}$; $P<0.0001$ ) (Figure 3c, left panel), serum titers of OVAspecific hzIgA were approximately threefold higher (Figure 3c, 

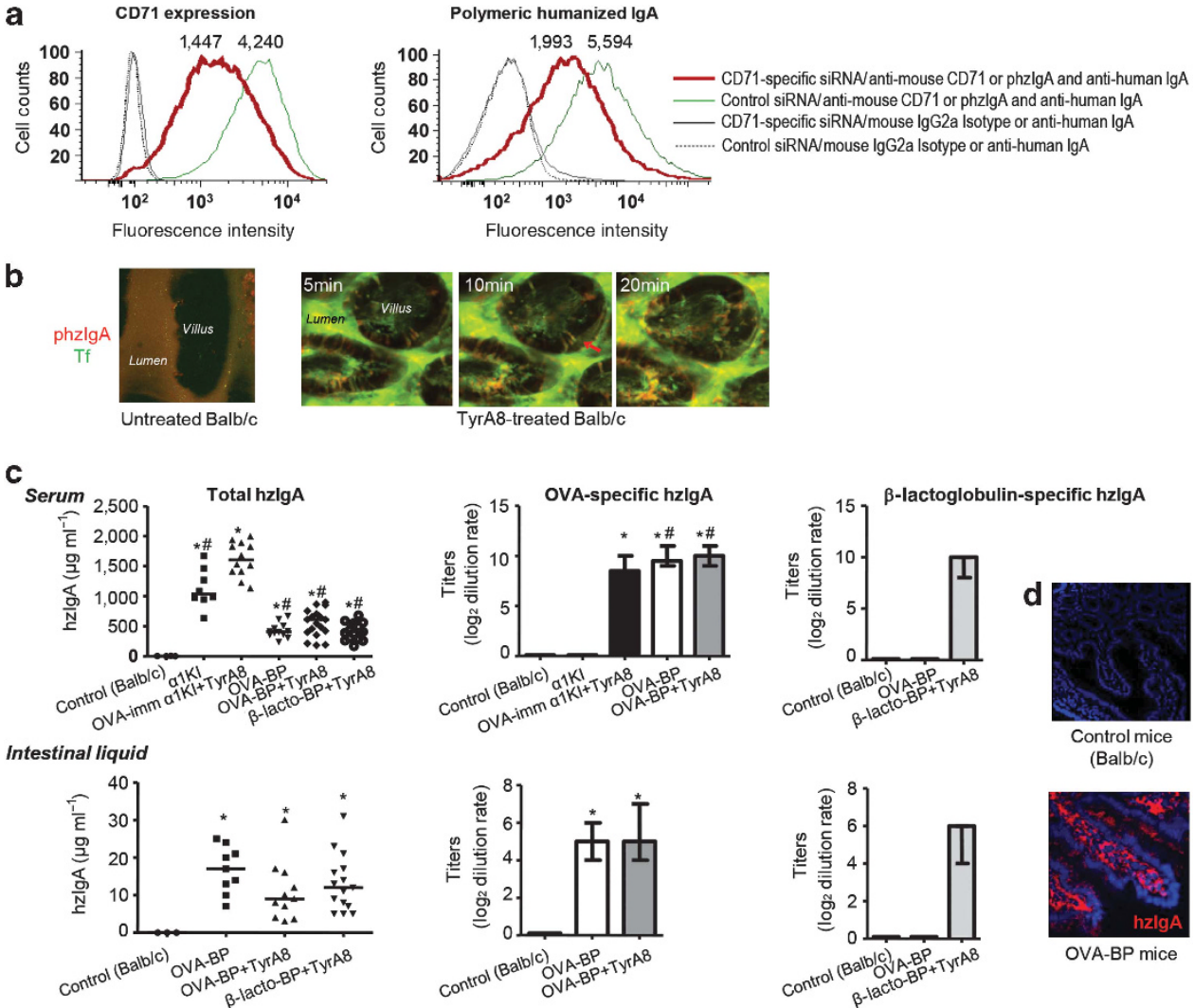

Figure 3 Distribution of humanized immunoglobulin $\mathrm{A}(\mathrm{hzlgA})$ in $\alpha 1 \mathrm{KI}$ mice and in mice carrying an $\alpha 1 \mathrm{KI}$-derived lgA-secreting backpack (BP) tumor. (a) Flow cytometry determination of CD71 expression (left panel) and binding of ovalbumin (OVA)-specific polymeric hzlgA (phzlgA; $300 \mu \mathrm{g} \mathrm{ml}{ }^{-1}$; right panel) in MODE K cells transfected with CD71-specific (red bold line) or control small interfering RNA (siRNA; green line). Median fluorescence intensities are indicated. (b) Analysis by multiphotonic video microscopy of apical binding and transcellular transport (arrow) of transferrin (Tf, green) and phzlgA (red) in duodenal segments from Balb/c mice either untreated (left panel) or $48 \mathrm{~h}$ after tyrphostin A8 (TyrA8) treatment (right panels) (see also Supplementary movies online). For the TyrA8-treated mice, images are shown 5, 10, and 20 min after the intraluminal injection of Tf and phzlgA. An image of a duodenal segment from a control mouse at the 20-min time point is shown. (c) Enzyme-linked immunosorbent assay determination of the concentrations of total hzlgA and of OVA-specific or $\beta$-lactoglobulin-specific hzlgA in serum (upper panel) and the intestinal lumen (lower panel) of control Balb/c mice, $\alpha 1 \mathrm{KI}$ mice immunized with OVA and treated or untreated with TyrA8 (OVA-imm $\alpha 1 \mathrm{KI}+\mathrm{TyrA} 8$ and $\alpha 1 \mathrm{KI}$, respectively) and in Balb/c mice carrying BP tumor secreting OVA-specific hzlgA (OVA-BP) or $\beta$-lactoglobulin-specific hzlgA ( $\beta$-lacto-BP) treated or not with TyrA8. Results are expressed as medians and ranges. *Significantly different from control Balb/c group $(P<0.003)$; ${ }^{\#}$ Significantly different from OVA-imm $\alpha 1 \mathrm{KI}+$ TyrA8 group $(P<0.003)$. (d) Confocal microscopy analysis of frozen duodenal sections from Balb/c mice untreated (control) or injected 2 weeks before with OVA-specific hzlgA-secreting hybridoma cells (OVA-BP) and stained with anti-human $\alpha$ chain labeled with Texas Red. Nuclei are stained in blue with DAPI (4,6-diamidino-2-phenylindole). One representative set of images of four comparable experiments is shown.

middle panel, median titers $\left(\log _{2}\right.$ dilution rate): 10.0 vs. 8.5 , $P<0.0013)$. As expected, anti-OVA-hzIgA or anti- $\beta$ lactoglobulin-hzIgA were exclusively found in the serum of mice bearing hybridoma cells specific for the respective antigen (Figure 3c, right panel). Moreover, OVA- or $\beta$-lactoglobulinspecific hzIgA were found in the intestinal fluid of the respective $\mathrm{BP}$ mice, attesting for the epithelial transport of hzIgA from the lamina propria into the gut lumen by the pIgR (Figure 3c, lower panels). Confocal microscopy of duodenal fragments confirmed the presence of hzIgA in the lamina propria of BP mice (Figure 3d), and western blot analysis demonstrated that the hzIgA purified from the intestinal fluid were pIgA containing the murine secretory component (SC) (see Supplementary Figure S1 online).

Consistent with SIgA-dependent protected transport in mice bearing OVA-specific BP tumor and treated with TyrA8 (OVA-BP + TyrA8 mice), the fraction of OVA transported in intact form in duodenal fragments mounted in Ussing chambers, detected by gel filtration chromatography and radio-detection, was significantly greater than in mice treated with TyrA8 but without BP tumor (control mice; $P<0.005$; Figure $4 \mathrm{~b}$, left panel, and Figure 4c). Accordingly, fluxes of intact OVA were 10-fold higher in OVA-BP + TyrA8 mice $\left(1.3 \mathrm{pmol} 90 \mathrm{~min}^{-1} \mathrm{~cm}^{-2}\right)$ than in control mice (0.1 pmol $90 \mathrm{~min}^{-1} \mathrm{~cm}^{-2}, \quad P<0.0001$ ) (Figure $\left.4 \mathbf{b}\right)$. In contrast, OVA was totally degraded during transport in the intestinal fragments of mice bearing OVA-specific BP tumor but not treated with TyrA8 (OVA-BP mice), a finding consistent with the dependence of SIgA-mediated protected transport on the apical expression of CD71 (Figure 4b). Arguing against non-specific paracellular leakage of intact OVA in mice treated with TyrA8, OVA total fluxes and transepithelial electrical resistance $(R)$ were similar in TyrA8treated and untreated mice, respectively (see Supplementary Figure S2 online). Furthermore, as previously described in an in vitro model, ${ }^{14}$ transport of intact protein required antigen- 

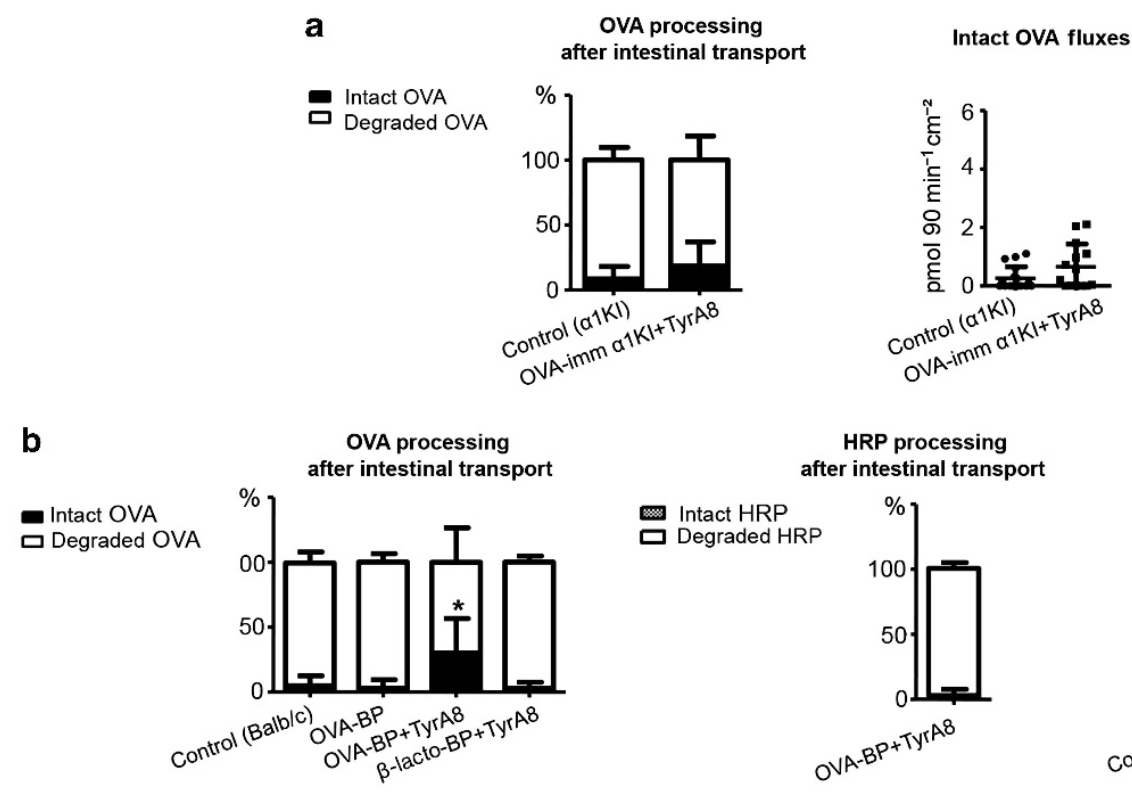

C

Control

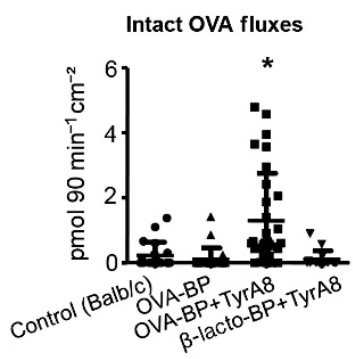

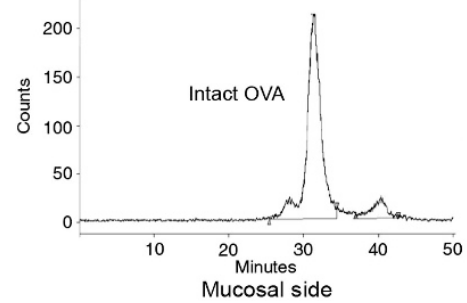

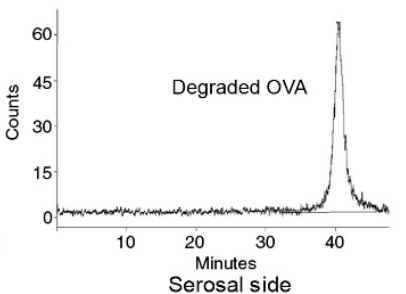

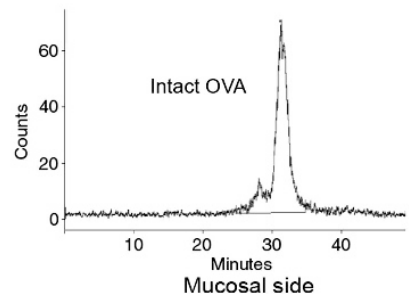

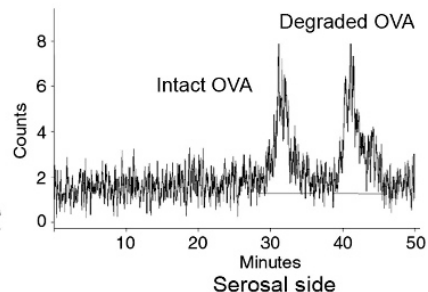

Figure 4 Intestinal transport of intact ovalbumin (OVA) in mice carrying a backpack (BP) tumor secreting OVA-specific humanized immunoglobulin A Analysis of the transport of ${ }^{3} \mathrm{H}$-OVA across duodenal fragments mounted in Ussing chambers (a) in OVA-immunized $\alpha 1 \mathrm{KI}$ mice treated with tyrphostin A8 (TyrA8) or (b) in Balb/c mice carrying OVA-BP or $\beta$-lactoglobulin-BP and treated or not with TyrA8. Percentages of intact (black bars) and degraded (white bars) ${ }^{3} \mathrm{H}$-OVA in the serosal compartment of intestinal fragments after transport are shown in the left panel. Fluxes (pmol $90 \mathrm{~min}^{-1} \mathrm{~cm}^{-2}$ ) of intact ${ }^{3} \mathrm{H}$-OVA transported from the mucosal-to-serosal compartment are shown in the right panel. Percentages of intact (black bars) and degraded (white bars) ${ }^{3} \mathrm{H}-\mathrm{HRP}$ (horseradish peroxidase) used as control in TyrA8-treated OVA-BP mice are shown in the middle panel. Results are expressed as means \pm s.d. and are from duplicate intestinal fragments from 8-12 $\alpha 1 \mathrm{KI}$ mice per group in panel $\mathbf{a}$ and from $12-19 \mathrm{Balb} / \mathrm{c}$ mice per group in panel $\mathbf{b}$. *Significantly higher in the OVA-BP + TyrA8 group $(P<0.003)$ compared with all the other groups. (c) Typical elution profiles of ${ }^{3} \mathrm{H}-\mathrm{OVA}$ after 90 min in Ussing chambers, measured by gel filtration chromatography of mucosal and serosal compartments in untreated Balb/c mice (Control) and in Balb/c mice carrying an OVA-BP tumor and treated by TyrA8 (OVA-BP mice + TyrA8).

specific SIgA. Thus, OVA was almost totally degraded during transport in TyrA8-treated mice producing $\beta$-lactoglobulinspecific IgA ( $\beta$-lacto-BP + TyrA8 mice) (Figure $4 \mathbf{b}$, left panel). Also consistent with the IgA-dependent transcellular transport of OVA and the lack of paracellular leakage, horseradish peroxidase (HRP) was almost totally degraded during transport across the epithelium of OVA-BP + TyrA 8 mice (Figure $4 \mathbf{b}$, middle panel). Taken together, these results indicate that TyrA8-treated mice bearing BP tumor secreting OVAspecific hzIgA ensure the CD71-mediated protected transport of SIgA complexed to OVA, thus recapitulating the process that we previously described for gluten peptides in CD.

\section{Transcytosis of IgA/OVA immune complexes activates OVA-specific CD4 ${ }^{+}$T lymphocytes in mesenteric lymph nodes (MLN) of D011.10 mice}

To assess whether IgA-mediated transcytosis might influence mucosal immune responses, hybridoma cells secreting OVAspecific hzIgA were grafted on day 0 onto DO11.10 mice with
$\mathrm{CD}^{+}{ }^{+} \mathrm{T}$ cells expressing a transgenic $\mathrm{T}$-cell receptor specific for OVA. Mice treated or not with TyrA8 on days 13 and 14 were gavaged with OVA on days 14,15 , and 16 . T-cell responses in the spleen and MLN were analyzed on day 20, before excessive growth of the BP tumor (see Supplementary Figure S3 online). No change in the activation markers or cytokine production of spleen cells was detected in either groups of mice. In contrast, a significant increase in the proportion of activated CD44 ${ }^{\text {hi }}$ CD62 $\mathrm{L}^{\text {low }}$ OVA-specific CD4 ${ }^{+}$T cells was observed in the MLN of mice gavaged with OVA (23-29\%), compared with sham-gavaged control mice (median 9.1\% (range 8-21), $P<0.02)$ ), indicating the specific activation of MLN T cells by dietary OVA. No further increase was observed in the OVABP TyrA8-treated group (see Supplementary Figure S4 online). A small increase in the percentage of Foxp $3^{+}$ OVA-specific $\mathrm{CD}^{+}{ }^{+} \mathrm{T}$ cells was also observed in OVA-fed mice compared with unfed animals and was only significant in mice without BP tumor (see Supplementary Figure S4 online). Strikingly, the expression of CD69, a molecule induced in 

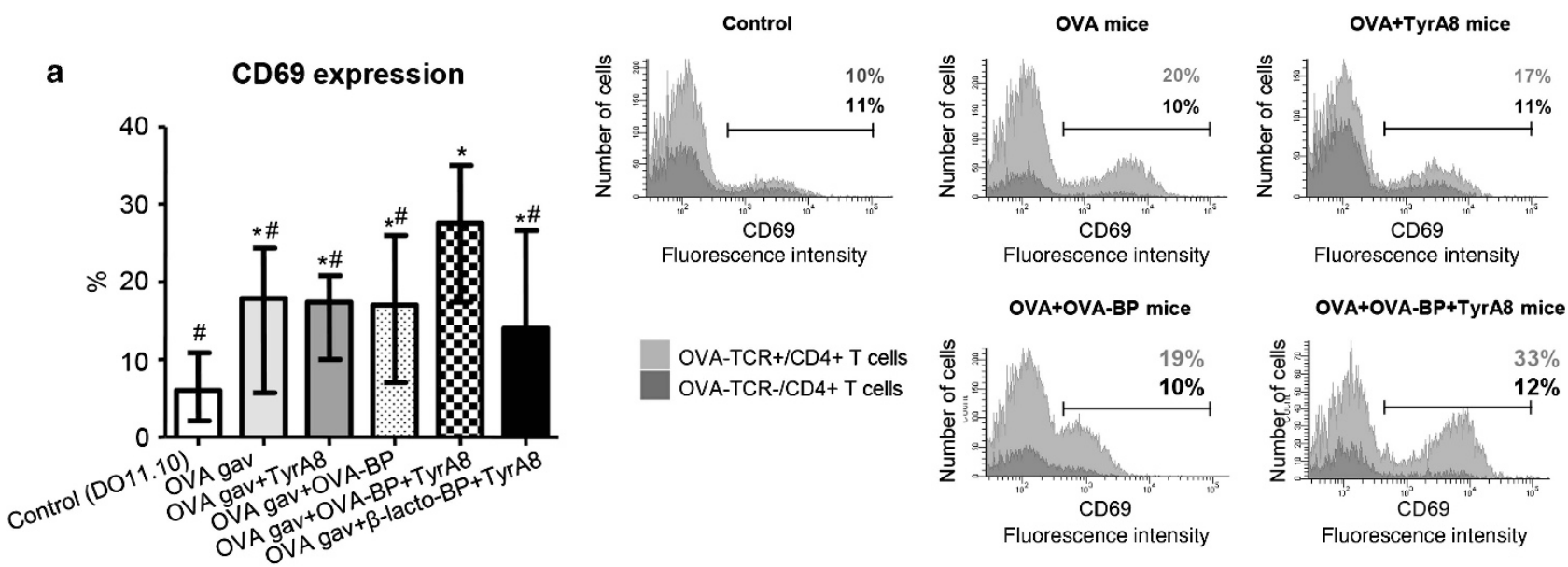

b OVA-induced secretion of IFN $\gamma$

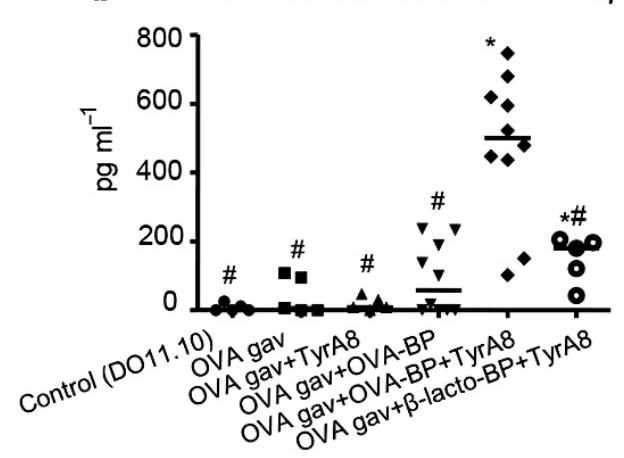

Figure 5 Enhanced activation of specific mesenteric lymph nodes (MLN) CD4 ${ }^{+}$T cells by immunoglobulin A-dependent intestinal transport of ovalbumin (OVA). (a) Flow cytometry analysis of CD69 expression by OVA-specific $\left(\mathrm{KJ1}_{1}-26^{+}\right.$) CD4 ${ }^{+} \mathrm{T}_{\text {cells }}$ and (b) enzyme-linked immunosorbent assay determination of interferon (IFN)- $\gamma$ secretion after a 72-h stimulation by OVA in MLN from DO11.10 transgenic mice daily gavaged with bicarbonate buffer (Control) or with $25 \mathrm{mg}$ OVA for 4 days (OVA gav) and bearing or not an OVA- or $\beta$-lactoglobulin backpack (BP) and treated or not with tyrphostin A8 (TyrA8). CD69 expression is shown for the various groups as histograms with medians and ranges (b, left panel). Flow cytometry analysis of one representative experiment out of four shows the percentage of $\mathrm{CD} 69^{+}$among $\mathrm{CD} 3^{+} \mathrm{CD} 4^{+} \mathrm{T}$ cells expressing (light grey) or not expressing (dark grey) the OVA-specific T-cell receptor (TCR; $\mathbf{a}$, right panel). Control and OVA gav groups: $n=7$ and 5 in panels a and $\mathbf{b}$, respectively; OVA gav + TyrA8 group: $n=5$ and 5; OVA gav + OVA-BP group: $n=10$ and 10; OVA gav + OVA-BP + TyrA8 group: $n=11$ and 10; OVA gav + $\beta$-lacto-BP + TyrA8 group: $n=7$ and 5. *Significantly different from the control group, $P<0.01$ in $\mathbf{a}, P<0.03$ in $\mathbf{b}$. "Significantly different from the OVA gav + OVA-BP + TyrA8 group, $P<0.01$ in panel $\mathbf{a}, P<0.03$ in panel $\mathbf{b}$.

activated $\mathrm{T}$ cells that negatively controls their egress from lymph nodes $^{21}$ and possesses immunomodulatory functions, ${ }^{22,23}$ was significantly increased in OVA-BP mice treated with TyrA8 (Figure 5a; $28 \%$ (17-35)), compared with the mice in all of the control groups, including: unfed controls (6\% (211), $P<0.0006)$, OVA-fed mice treated or untreated with TyrA8 (18\% (6-24), $P<0.003$ and $17 \%(10-21), P<0.011$, respectively), and OVA-fed mice carrying a $\beta$-lactoglobulinspecific BP tumor and treated with TyrA8 (14\% (10-27), $P<0.005)$. To assess the regulatory vs. pro-inflammatory effect of the OVA-specific $\mathrm{T}$ cells elicited in the different groups, production of interferon (IFN) $-\gamma$ and interleukin (IL)-10 was measured in the supernatants of MLN cells stimulated in vitro with OVA for $72 \mathrm{~h}$. No significant IL-10 production was observed in either groups (see Supplementary Figure S4 online). In contrast, a significant increase in OVA-induced IFN- $\gamma$ secretion was observed in OVA-fed mice carrying an OVA-BP tumor and treated with TyrA8 compared with all the other groups of mice, including mice carrying OVA-BP tumor but not treated with TyrA8 and TyrA8-treated mice carrying the irrelevant anti- $\beta$-lactoglobulin BP tumor (Figure 5b). These results indicate that the IgA-mediated transcytosis of OVA promotes activation of OVA-specific $\mathrm{T}$ cells producing IFN- $\gamma$, a feature of a Thelper type 1 skew often accompanying pro-inflammatory reactions.

\section{DISCUSSION}

Following our previous ex vivo and in vitro studies in $\mathrm{CD}$ showing that abnormal targeting of the $\mathrm{CD} 71$ receptor to the apical surface of enterocytes induced a protected apico-basal transport of dietary antigens bound to specific SIgA, we have established a mouse model that corroborates these findings. Using this model, we provide evidence that this mechanism can stimulate the response of mucosal $\mathrm{CD} 4{ }^{+} \mathrm{T}$ cells against the transported antigen and promote their production of IFN- $\gamma$. Retro-transcytosis of IgA-gliadin complexes may thus foster abnormal intestinal T-cell activation in human pathological 
conditions associated with the ectopic expression of CD71 at the gut surface, as observed in $\mathrm{CD}$.

CD71 is best known as a high-affinity receptor for transferrin, but it also has a role as a low-avidity receptor for pIgA1 in humans. ${ }^{12-14} \mathrm{~A}$ recent report by Papista et al. ${ }^{24}$ based on co-localization studies in mouse intestine, suggested that murine IgA might bind to murine CD71. However, using flow cytometry, we were unable to demonstrate any significant binding of murine $\mathrm{pIgA}$ to $\mathrm{CD} 71^{+}$murine epithelial cell lines. The latter result is consistent with the fact that murine IgA are structurally close to human IgA2 which, in contrast to human IgA1, does not bind CD71, possibly due to their truncated hinge region. ${ }^{13,25}$ In keeping with recent studies, ${ }^{20,26}$ we confirmed that murine CD71, which bears strong homology to human CD71, can bind human SIgA and phzIgA1. Moreover, by applying the Duolink technique to MODE K cells, we showed that murine CD71, like human CD71, ${ }^{14}$ can interact directly with human SIgA. In order to set up an in vivo model of CD71mediated absorption of SIgA immune complexes in mice, we therefore turned to the recently described transgenic $\alpha 1 \mathrm{KI}$ mouse, which produces hzIgA, made of human IgA1 heavy chains and murine kappa light chains. HzIgA can bind the murine $J$ chain and form dimeric IgA, which is secreted into the intestinal lumen via the murine $\mathrm{pIgR} .{ }^{27}$ Using murine epithelial cell lines, we show that, like human IgA1, phzIgA bound murine CD71. In $\alpha 1 \mathrm{KI}$ mice immunized with OVA, OVAspecific IgA were detected in substantial amounts in the serum but were undetectable in the intestinal fluid. In order to obtain high concentrations of antigen-specific IgA in the intestinal fluid, we therefore used the BP tumor technique, which has already been used to demonstrate that specific SIgA delivered into the intestinal lumen can protect against intestinal pathogens. ${ }^{28,29}$ Consistent with these studies, significant amounts of OVA-specific SIgA were detected in the intestinal fluid of Balb/c mice grafted with hybridoma cells secreting OVA-specific humanized $\alpha 1 \mathrm{KI}$ IgA.

We have previously reported that CD71-mediated retrotransport of SIgA immune complexes across the intestinal epithelium depends on the overexpression and redistribution of CD71 from the basolateral to the apical membrane of enterocytes. ${ }^{12,14}$ Interestingly, previous studies have established that CD71-mediated apical-to-basal transport of antigens coupled to transferrin can be observed in the intestine of rats treated with TyrA8, a GTPase inhibitor with little toxicity. ${ }^{19}$ The mechanism has not been elucidated, but in vitro studies in Caco- 2 cells showed that TyrA8 stimulated recycling through Rab11-rich compartments. ${ }^{18}$ Here, we observed that intestinal epithelial expression of CD71 was markedly upregulated in mice treated with TyrA8 and was detected at both the basal and apical poles of enterocytes as is observed in ACD. Moreover, as the binding sites of $\mathrm{pIgA}$ and transferrin to CD71 have been shown to be distinct, ${ }^{20}$ multiphotonic confocal video microscopy could be used to follow-up and demonstrate rapid and simultaneous apico-basal transepithelial transport of transferrin and of either human or phzIgA in intestinal segments of TyrA8-treated mice but not in segments from untreated mice.
We further used TyrA8 treatment to test the role of SIgA in the transport of dietary OVA in OVA-immunized $\alpha 1 \mathrm{KI}$ mice or in OVA-BP mice. Confirming that this mechanism can deliver intact dietary proteins or peptides into the lamina propria, transepithelial transport of intact OVA could be demonstrated in duodenal samples from TyrA8-treated OVA-BP mice but not in samples from untreated mice or from mice bearing a control BP tumor secreting $\beta$-lactoglobulin-specific hzIgA. Consistent with a mandatory role for luminal SIgA specific for the transported antigen, no significant transport could be demonstrated in the duodenum of OVA-immunized $\alpha 1 \mathrm{KI}$ mice, which, in contrast to OVA-BP mice, did not show detectable concentrations of OVA-specific IgA in the intestinal fluid. Moreover, no transport of intact HRP was observed in TyrA8-treated OVA-BP mice. This observation, together with unchanged transepithelial electrical resistance, provides strong evidence against non-specific paracellular leakage of proteins. These results also indicate, as previously reported in vitro, ${ }^{14}$ that SIgA-dependent transport of proteins is antigen-specific.

We next examined how the abnormal apico-basal transport of intact dietary OVA by SIgA induced in TyrA8-treated DO11.10 mice carrying an OVA-BP tumor might influence the local $\mathrm{T}$-cell response. Indeed, previous studies have shown that IgE- and IgG-dependent transport of luminal antigens across intestinal epithelium can stimulate local allergic ${ }^{30,31}$ or pro-inflammatory ${ }^{16,17}$ immune responses, respectively. In untreated DO11.10 mice, OVA feeding stimulated the appearance of activated $\mathrm{CD} 44^{\mathrm{hi}} \mathrm{CD} 62 \mathrm{~L}^{\mathrm{lo}}$ cells and a small but significant increase in the proportion of Foxp $3^{+}$cells among MLN OVA-specific CD $4^{+} \mathrm{T}$ cells, a finding consistent with previous reports and with the induction of tolerance to this dietary antigen. ${ }^{32,33}$ Accordingly, MLN cells from OVA-fed mice did not produce a significant amount of IFN- $\gamma$ in response to OVA. A comparable increase in the number of CD $44^{\text {hi }}$ CD62 $\mathrm{L}^{\text {lo }}$ OVA-specific $\mathrm{CD} 4{ }^{+} \mathrm{T}$ cells was observed in the MLN of TyrA8-treated DO11.10 mice carrying an OVA-BP tumor but the increase in Foxp3 ${ }^{+} \mathrm{T}$ cells was not significant. In contrast, the latter cells displayed significantly higher expression of the activation marker CD69 than cells from the control mice and notably than cells from TyrA8-treated mice bearing or not an irrelevant BP tumor. Moreover, MLN lymphocytes from TyrA8-treated DO11.10 mice bearing the OVA-specific BP tumor produced significantly higher amounts of the proinflammatory cytokine IFN- $\gamma$ in response to OVA than MLN lymphocytes from control mice. In the present model, the requirement for a hybridoma $\mathrm{BP}$ to obtain sufficient amounts of specific hzIgA in the intestinal lumen precluded long-term experiments to assess possible consequences on gut homeostasis. Despite this limitation, the obtained results strongly suggest that the abnormal SIgA-mediated transport of dietary antigen can foster local T-cell activation and compromise the establishment of oral tolerance. One important question is how IgA, known to have a central role in maintaining intestinal homeostasis, may trigger a paradoxical pro-inflammatory response. Recent work has demonstrated that CD71, besides its well-known role in endocytosis, can, upon binding to pIgA, 
initiate a signalling cascade that promotes both cell proliferation and the release of pro-inflammatory cytokines. ${ }^{20}$ Notably in IgA nephropathy, the binding of pIgA to CD71 abnormally expressed by mesangial cells in the glomeruli can trigger the ERK (extracellular signal-regulated kinase)-mediated release of IL-6. ${ }^{34}$ Future studies, which are in progress, will aim at defining whether a comparable scenario operates in the gut epithelium of patients with ectopic surface expression of CD71 and participates in the development or exacerbation of intestinal inflammation in CD or in other conditions associated with intestinal CD71 upregulation, such as iron deficiency and epithelial repair. Together, these results provide a new perspective of how a key immune mechanism of defence can be turned into a threat in pathological conditions.

\section{METHODS}

Mice. Adult female Balb/c and Balb/c DO11.10 transgenic mice with OVA-specific $\mathrm{CD}^{+}{ }^{+} \mathrm{T}$ cells were obtained from CNRS-Orléans (Orleans, France) and Jackson ImmunoResearch Europe (Marseille, France), respectively. Balb/c $\alpha 1 \mathrm{KI}$ mice, which produce hzIgA made of human $\alpha 1$ heavy chains and murine light chains, ${ }^{27}$ were handled by $B$ Cell Design (Limoges, France). After immunization by intraperitoneal injections of $10 \mu \mathrm{g}$ OVA or $\beta$-lactoglobulin, initially delivered in complete Freund's adjuvant and then delivered three times at weekly intervals in incomplete Freund's adjuvant, $\alpha 1 \mathrm{KI}$ mice were either gavaged with denaturated OVA $(50 \mu \mathrm{g})$ twice a week for 2 weeks or used to obtain SP2/0-derived hybridoma cells producing specific hzIgA according to Kohler and Milstein. ${ }^{35}$ To generate BP tumors secreting specific hzIgA, $2.5 \times 10^{6}$ hybridoma cells were injected subcutaneously into the upper back of $8-10$-week-old female Balb/c or DO11.10 mice as previously described. ${ }^{36}$ In some experiments, mice were injected intraperitoneally with TyrA $8\left(12 \mathrm{mg} \mathrm{kg}^{-1}\right.$, Covalab, Villeurbanne, France) twice, one day apart. This study was approved by the local ethics committee for animal experimentation.

IgA binding and CD71 siRNA transfection of murine epithelial cell lines. MODE $\mathrm{K}$ cells (kindly provided by $\mathrm{D}$. Kaiserlian) were transfected with 20 nM of Alexa 488-labeled murine CD71 siRNA MmTfrc 5 or control-Alexa 488-labeled siRNA (Qiagen, SAS, Courtaboeuf, France) for $96 \mathrm{~h}$ according to the manufacturer's instructions. After blocking Fc receptors with rat anti-mouse CD16/CD32 $\left(100 \mu \mathrm{g} \mathrm{ml}^{-1}\right.$, BD Biosciences, Le Pont-de-Claix, France) for $15 \mathrm{~min}$ at $4{ }^{\circ} \mathrm{C}$, cells were labelled with PE (phycoerythrin)-labelled rat anti-mouse CD71 or control IgG2a $\left(10 \mu \mathrm{g} \mathrm{ml}^{-1}\right.$; BD Biosciences) for $20 \mathrm{~min}$ and/or incubated for $1 \mathrm{~h}$ at $4{ }^{\circ} \mathrm{C}$ with indicated concentrations of murine, human, or hzIgA. Murine pIgA (containing the J chain but not the SC) were produced and purified from the HNK20 hybridoma cell clone. ${ }^{37}$ Purified human colostral SIgA were obtained from a commercial source (Fitzgerald Industries, Acton, MA). Anti-OVA hzIgA were purified from the supernatant of anti-OVA hybridoma cultures using peptide $M$, a high avidity and specific ligand for human IgA, ${ }^{38}$ coupled to agarose beads (Cayla-Invivogen, Toulouse, France) according to the manufacturer's instructions. For binding studies, polymeric anti-OVA hzIgA (approximately 50\% after purification of hybridoma supernatant by peptide $\mathrm{M}$ /agarose) were then separated from monomeric hzIgA by size exclusion chromatography. As shown in Supplementary Figure $\mathbf{S 1}$ online, polymeric anti-OVA hzIgA contained the murine J chain. IgA binding was revealed using two successive 20-min incubations with biotinylated anti-mouse $\alpha$ chain goat IgG $\left(25 \mu \mathrm{g} \mathrm{ml}^{-1}\right.$; CliniSciences, Nanterre, France) or biotinylated goat anti-human $\alpha$ chain $\mathrm{F}\left(\mathrm{ab}^{\prime}\right)_{2} \quad\left(50 \mu \mathrm{g} \mathrm{ml}^{-1}\right.$; Southern Biotech, Birmingham, AL), followed by APC (allophycocyanin) streptavidin ( $4 \mu \mathrm{g} \mathrm{ml}^{-1}$; BD Biosciences). Cells were analyzed on a BD FACS Canto flow cytometer using Flow-Jo software for data processing (BD Biosciences).

Confocal microscopy. Acetone-fixed sections $(8 \mu \mathrm{m})$ of frozen mouse duodenum were labelled with FITC rat anti-mouse CD71 IgG2a or isotype control (Caltag Laboratories, Burlingame, CA) for $90 \mathrm{~min}$. CD71-SIgA interactions were analyzed on MODE K cells using CD71 $\mathrm{mAb}$ (Life Technologies, Courtaboeuf, France) and rabbit anti-human IgA (Abcam, Paris, France) and the Duolink in situ Proximity Ligation Assay (PLA; OLINK Biosciences, Uppsala Sweden) as previously described. ${ }^{14}$ Labeling was analyzed with a TCS SP5 confocal microscope (Leica, Mannheim, Germany) using CLSM software.

Multiphotonic video microscopy. Duodenal segments from mice treated or not with TyrA8 were maintained on ice and injected with $50 \mu \mathrm{g} \mathrm{ml}^{-1}$ human holotransferrin/Alexa fluor 488 (Molecular Probes, Eugene, OR) and with $250 \mu \mathrm{g} \mathrm{ml}^{-1}$ of either human SIgA or phzIgA previously labelled with the red fluoroprobe 547 according to the manufacturer's instructions (Molecular Probes). Intestinal segments were then opened along the mesenteric border, extensively washed with phosphate-buffered saline at $4{ }^{\circ} \mathrm{C}$ and placed into a perfusion POC-air chamber (PeCon Gmbh, Erbach, Germany) that was inserted in the $37^{\circ} \mathrm{C}$ incubator of a multiphoton microscope (TriM Scope, LaVision BioTec $\mathrm{GmbH}$, Guyancourt, France). Internalization of SIgA and transferrin was monitored, using the time-lapse program of Inspector Pro software (Bielefeld, Germany), every $60 \mathrm{~s}$ for $20 \mathrm{~min}$. Images were post-treated with Image J software (NIH, Bethesda, MD) and the StackReg plugin (NIH).

Intestinal transport of ${ }^{3} \mathrm{H}-\mathrm{OVA}$. Mouse duodenal fragments were mounted in Ussing chambers and ${ }^{3} \mathrm{H}-\mathrm{OVA}\left(30 \mu \mathrm{g} \mathrm{ml}^{-1}\right)$ was added to the mucosal compartment. After $90 \mathrm{~min}$, OVA-derived fragments were analyzed in the mucosal and serosal compartments using Superdex 200 gel filtration chromatography (Interchim, Montluçon, France) and radio-detection, as described previously. ${ }^{12}$ Results were expressed as percentages (or fluxes) of intact OVA after transport into the serosal compartment. Tissue viability was checked by monitoring electrical parameters using an automatic clamping device (World Precision Instruments, Hertfordshire, UK), as described. ${ }^{12}$

Immunological analyses. Concentrations of total and OVA-specific hzIgA in the blood and intestinal fluid were assessed by enzyme-linked immunosorbent assay using Immulon 2HB 96-well plates (VWR, Fontenay-sous-Bois, France) coated overnight with $5 \mu \mathrm{g} \mathrm{ml}^{-1}$ mouse anti-human IgA (Abcam) or with $1 \mu \mathrm{g} \mathrm{ml}^{-1}$ of OVA (Grade V, SigmaAldrich Chimie, Lyon, France), respectively. Binding of IgA was revealed with $\mathrm{HRP}$-conjugated murine anti-human $\operatorname{IgA}(1 / 8000$, Abcam). Polymeric and monomeric hzIgA in serums and intestinal fluids were separated onto 4-15\% polyacrylamide gradient gels run in sodium dodecyl sulfate (Bio-Rad, Hercules, CA) in non-reducing conditions and analyzed by western blot assays using anti-human $\alpha$ chain goat antiserum (Beckman-Coulter, Jersey City, NJ). The presence of the murine J chain or SC was studied in hzIgA purified with peptide $\mathrm{M}$ /agarose from anti-OVA hybridoma supernatant, or from serums and intestinal fluids of mice grafted with the OVA-BP tumor, by western blot assays in reducing conditions using rabbit anti-mouse J chain (Santa Cruz Biotechnologies, Santa Cruz, CA) and rabbit antimouse SC, ${ }^{39}$ respectively. Mucosal T-cell responses were assessed in DO11.10 transgenic mice with or without a BP tumor of hybridoma cells secreting OVA- or $\beta$-lactoglobulin-specific hzIgA. CD71 expression was induced by administration of TyrA8 on days 13 and 14 after injection of hybridoma cells. Mice were gavaged on days 14, 15, and 16 with $25 \mathrm{mg}$ OVA and killed 4 days later (see Supplementary Figure S3 online). Spleen and MLN cells were membrane-labelled with FITC-KJ1-26 mAb specific for the DO11.10 OVA-specific T-cell receptor (Life Technologies), e-Fluor anti-CD45 (30-F11), PE-Cy7 anti-CD3 (145-2C11), APC-H7 anti-CD4 (GK1.5), PE anti-CD44 (IM7), APC anti-CD62L (MEL-14), and biotinylated hamster antiCD69 (H1.2F3) or control isotypes for $20 \mathrm{~min}$ at $4{ }^{\circ} \mathrm{C}$. Biotin was detected using PerCP streptavidin (BD Biosciences). For intracellular 
Foxp3 staining, membrane-labelled cells were fixed and permeabilized using the Foxp3 Staining Buffer Set and incubated with PeCy5 antiFoxp3 (FJK-16s) (all from eBioscience SAS, Paris, France) or control isotype (BD Biosciences) for $30 \mathrm{~min}$ at $4{ }^{\circ} \mathrm{C}$. Staining was analyzed on a BD FACSCanto flow cytometer using FACSDIVA software (BD Biosciences). For cytokine production, spleen and MLN cells were cultured in 96-well round bottom plates (BD Biosciences) in RPMI supplemented with $10 \%$ fetal calf serum, $1 \mathrm{~mm}$ non-essential amino acids, $1 \%$ penicillin and streptomycin (Life Technologies), and $5 \times 10^{-5} \mathrm{M} \beta$-mercapto-ethanol (Bio-Rad Laboratories, Marne La Coquette, France) and were either stimulated with OVA $\left(0.5 \mathrm{mg} \mathrm{ml}^{-1}\right)$ for 3 days or not stimulated. IFN- $\gamma$ and IL-10 secretion was analyzed using specific enzyme-linked immunosorbent assays according to the manufacturer's instructions (R\&D Systems Europ Ltd, Lille, France).

Statistics. Statistical analysis was carried out using the GraphPad Prism software (Ritme, Paris, France). Results are expressed as means \pm s.d. for transport/processing studies and as medians with ranges for all other experiments. Comparisons among all groups were performed using the non-parametric Mann-Whitney $U$ test and considered significant at $P<0.05$.

SUPPLEMENTARY MATERIAL is linked to the online version of the paper at http://www.nature.com/mi

\section{ACKNOWLEDGEMENTS}

This work was supported by the French National Research Agency (ANR-ALIA-017-01), the Princess Grace de Monaco Foundation and INSERM. JA was supported by ANR and by a research grant from BSL Behring. The laboratory of BC is funded by grant No. 3100-138422 from the Swiss Science Research Foundation.

\section{AUTHOR CONTRIBUTIONS}

$\mathrm{JA}, \mathrm{CL}, \mathrm{BM}, \mathrm{CD}, \mathrm{MGT}, \mathrm{NC}-\mathrm{B}$, and MH contributed to study design and revised the manuscript. JA, CL, GC, AC, and MC participated in data acquisition and analysis; $B C$ provided reagents and revised the manuscript; JA, MH, and NC-B wrote the manuscript; $\mathrm{MH}$ and NC-B supervised the study.

\section{DISCLOSURE}

The authors declared no conflict of interest.

(c) 2014 Society for Mucosal Immunology

\section{REFERENCES}

1. Menard, S., Cerf-Bensussan, N. \& Heyman, M. Multiple facets of intestinal permeability and epithelial handling of dietary antigens. Mucosal Immunol. 3, 247-259 (2010).

2. Matysiak-Budnik, T. et al. Alterations of the intestinal transport and processing of gliadin peptides in celiac disease. Gastroenterology 125, 696-707 (2003).

3. Fasano, A. et al. Zonulin, a newly discovered modulator of intestinal permeability, and its expression in coeliac disease. Lancet 355, 1518-1519 (2000).

4. Menzies, I.S. Intestinal permeability in coeliac disease. Gut 13, 847 (1972).

5. Oberhuber, G. \& Vogelsang, H. Gastrointestinal permeability in celiac disease. Gastroenterology 114, 226 (1998).

6. Schulzke, J.D., Bentzel, C.J., Schulzke, I., Riecken, E.O. \& Fromm, M. Epithelial tight junction structure in the jejunum of children with acute and treated celiac sprue. Pediatr. Res. 43, 435-441 (1998).

7. Schulzke, J.D., Schulzke, I., Fromm, M. \& Riecken, E.O. Epithelial barrier and ion transport in coeliac sprue: electrical measurements on intestinal aspiration biopsy specimens. Gut 37, 777-782 (1995).

8. Menard, S. et al. Paracellular versus transcellular intestinal permeability to gliadin peptides in active celiac disease. Am. J. Pathol. 180, 608-615 (2012).
9. Zimmer, K.P., Poremba, C., Weber, P., Ciclitira, P.J. \& Harms, E. Translocation of gliadin into HLA-DR antigen containing lysosomes in coeliac disease enterocytes. Gut 36, 703-709 (1995).

10. Schumann, M. et al. Mechanisms of epithelial translocation of the alpha(2)gliadin-33mer in coeliac sprue. Gut 57, 747-754 (2008).

11. Bethune, M.T., Ribka, E., Khosla, C. \& Sestak, K. Transepithelial transport and enzymatic detoxification of gluten in gluten-sensitive rhesus macaques. Plos One 3, e1857 (2008).

12. Matysiak-Budnik, T. et al. Secretory lgA mediates retrotranscytosis of intact gliadin peptides via the transferrin receptor in celiac disease. J. Exp. Med. 205, 143-154 (2008).

13. Moura, I.C. et al. Glycosylation and size of $\lg A 1$ are essential for interaction with mesangial transferrin receptor in IgA nephropathy. J. Am. Soc. Nephrol. 15, 622-634 (2004).

14. Lebreton, C. et al. Interactions among secretory immunoglobulin A, CD71, and transglutaminase-2 affect permeability of intestinal epithelial cells to gliadin peptides. Gastroenterology 143, 698-707. e691-694 (2012).

15. Rauhavirta, T. et al. Epithelial transport and deamidation of gliadin peptides: a role for coeliac disease patient immunoglobulin A. Clin. Exp. Immunol. 164, 127-136 (2011).

16. Yoshida, M. et al. Human neonatal Fc receptor mediates transport of IgG into luminal secretions for delivery of antigens to mucosal dendritic cells. Immunity 20, 769-783 (2004).

17. Yoshida, M. et al. Neonatal Fc receptor for lgG regulates mucosal immune responses to luminal bacteria. J. Clin. Invest. 116, 2142-2151 (2006).

18. Norouziyan, F., Shen, W.C. \& Hamm-Alvarez, S.F. Tyrphostin A8 stimulates a novel trafficking pathway of apically endocytosed transferrin through Rab11-enriched compartments in Caco-2 cells. Am. J. Physiol. Cell Physiol. 294, C7-21 (2008).

19. Xia, C.Q. \& Shen, W.C. Tyrphostin-8 enhances transferrin receptormediated transcytosis in Caco-2- cells and inreases hypoglycemic effect of orally administered insulin-transferrin conjugate in diabetic rats. Pharm. Res. 18, 191-195 (2001).

20. Coulon, S. et al. Polymeric lgA1 controls erythroblast proliferation and accelerates erythropoiesis recovery in anemia. Nat. Med. 17, 1456-1465 (2011).

21. Shiow, L.R. et al. CD69 acts downstream of interferon-alpha/beta to inhibit S1P1 and lymphocyte egress from lymphoid organs. Nature 440, 540-544 (2006).

22. Sancho, D. et al. CD69 downregulates autoimmune reactivity through active transforming growth factor-beta production in collagen-induced arthritis. J. Clin. Invest. 112, 872-882 (2003).

23. Miki-Hosokawa, T. et al. CD69 controls the pathogenesis of allergic airway inflammation. J. Immunol. 183, 8203-8215 (2009).

24. Papista, C. et al. Gluten induces coeliac-like disease in sensitised mice involving IgA, CD71 and transglutaminase 2 interactions that are prevented by probiotics. Lab. Invest. 92, 625-635 (2012).

25. Tucker, P.W., Slightom, J.L. \& Blattner, F.R. Mouse IgA heavy chain gene sequence: implications for evolution of immunoglobulin hinge axons. Proc. Natl. Acad. Sci. USA 78, 7684-7688 (1981).

26. Berthelot, L. et al. Transglutaminase is essential for IgA nephropathy development acting through IgA receptors. J. Exp. Med. 209, 793-806 (2012).

27. Duchez, S. et al. Premature replacement of mu with alpha immunoglobulin chains impairs lymphopoiesis and mucosal homing but promotes plasma cell maturation. Proc. Natl. Acad. Sci. USA 107, 3064-3069 (2010).

28. Michetti, P., Mahan, M.J., Slauch, J.M., Mekalanos, J.J. \& Neutra, M.R. Monoclonal secretory immunoglobulin A protects mice against oral challenge with the invasive pathogen Salmonella typhimurium. Infect. Immun. 60, 1786-1792 (1992).

29. Hutchings, A.B. et al. Secretory immunoglobulin A antibodies against the sigma1 outer capsid protein of reovirus type 1 Lang prevent infection of mouse Peyer's patches. J. Virol. 78, 947-957 (2004).

30. Yang, P.C., Berin, M.C., Yu, L.C., Conrad, D.H. \& Perdue, M.H. Enhanced intestinal transepithelial antigen transport in allergic rats is mediated by lgE and CD23 (FcepsilonRII). J. Clin. Invest. 106, 879-886 (2000).

31. Bevilacqua, C. et al. Food allergens are protected from degradation during CD23-mediated transepithelial transport. Int. Arch Allergy Immunol. 135, 108-116 (2004). 


\section{ARTICLES}

32. Hauet-Broere, F., Unger, W.W., Garssen, J., Hoijer, M.A., Kraal, G. \& Samsom, J.N. Functional CD25- and CD25 + mucosal regulatory T cells are induced in gut-draining lymphoid tissue within $48 \mathrm{~h}$ after oral antigen application. Eur. J. Immunol. 33, 2801-2810 (2003).

33. Hadis, U. et al. Intestinal tolerance requires gut homing and expansion of FoxP3 + regulatory T cells in the lamina propria. Immunity $34,237-246$ (2011).

34. Tamouza, $\mathrm{H}$. et al. The IgA1 immune complex-mediated activation of the MAPK/ERK kinase pathway in mesangial cells is associated with glomerular damage in IgA nephropathy. Kidney Int. 82, 1284-1296 (2012).

35. Kohler, G. \& Milstein, C. Continuous cultures of fused cells secreting antibody of predefined specificity. Nature 256, 495-497 (1975).

36. Winner, L. 3rd, Mack, J., Weltzin, R., Mekalanos, J.J., Kraehenbuhl, J.P. \& Neutra, M.R. New model for analysis of mucosal immunity: intestinal secretion of specific monoclonal immunoglobulin A from hybridoma tumors protects against Vibrio cholerae infection. Infect. Immun. 59, 977982 (1991).

37. Favre, L., Spertini, F. \& Corthésy, B. Secretory IgA possesse intrinsic modulatory properties stimulating mucosal and systemic immune responses. J. Immunol. 175, 2793-2800 (2005).

38. Pleass, R.J., Areschoug, T., Lindahl, G. \& Woof, J.M. Streptococcal IgAbinding proteins bind in the Calpha 2-Calpha 3 interdomain region and inhibit binding of IgA to human CD89. J. Biol. Chem. 276, 8197-8204 (2001).

39. Crottet, P, Cottet, S \& Corthésy, B. Expression, purification and biochemical characterization of recombinant murine secretory component: a novel tool in mucosal immunology. Biochem. J. 341, 299-306 (1999). 